\title{
sciendo
}

\section{Reaction to a Visual Stimulus: Anticipation with Steady and Dynamic Contractions}

\author{
by \\ Agostina Casamento-Moran ${ }^{1}$, Stefan Delmas ${ }^{1}$, Seoung Hoon Park ${ }^{1}$, Basma Yacoubi ${ }^{1}$, \\ Evangelos A. Christou ${ }^{1}$
}

\begin{abstract}
Reacting fast to visual stimuli is important for many activities of daily living and sports. It remains unknown whether the strategy used during the anticipatory period influences the speed of the reaction. The purpose of this study was to determine if reaction time (RT) differs following a steady and a dynamic anticipatory strategy. Twenty-two young adults (21.0 \pm 2.2 yrs, 13 women) participated in this study. Participants performed 15 trials of a reaction time task with ankle dorsiflexion using a steady (steady force at 15\% MVC) and a dynamic (oscillating force from 10-20\% $M V C$ ) anticipatory strategy. We recorded primary agonist muscle (tibialis anterior; TA) electromyographic (EMG) activity. We quantified $R T$ as the time interval from the onset of the stimulus to the onset of force. We found that a dynamic anticipatory strategy, compared to the steady anticipatory strategy, resulted in a longer $R T(p=0.04)$. We classified trials of the dynamic condition based on the level and direction of anticipatory force at the moment of the response. We found that $R T$ was longer during the middle descending relative to the middle ascending and the steady conditions $(p<0.01)$. All together, these results suggest that $R T$ is longer when preceded by a dynamic anticipatory strategy. Specifically, the longer $R T$ is a consequence of the variable direction of force at which the response can occur, which challenges the motor planning process.
\end{abstract}

Key words: reaction time, anticipation, steady contraction, sinusoidal contraction.

\section{Introduction}

Reacting fast to visual stimuli is important for many activities of daily living and sports. In soccer, for example, the goalkeeper must react fast to save a penalty kick. The goalkeeper can anticipate a penalty kick either by remaining motionless or oscillating. Thus, an anticipatory strategy can be either steady or dynamic. However, it remains unknown whether the strategy used in anticipation to a stimulus influences the speed of the reaction. Here, we examine whether reaction time (RT) differs after a steady and a dynamic anticipatory strategy.

Reaction time is the time interval between the stimulus onset and the onset of the response and reflects the speed at which the nervous system processes the stimulus and executes a response
(Schmidt and Lee, 2019). RT primarily consists of two components: premotor time and electromechanical delay (EMD) (Schmidt and Lee, 2019). Premotor time is the temporal interval from stimulus onset to the onset of muscle activity (Botwinick and Thompson, 1966; Schmidt and Lee, 2019) and represents the time it takes to identify the stimulus, process it, and generate a response (i.e. information processing). EMD is the temporal interval from the onset of muscle activity to the onset of force (Botwinick and Thompson, 1966; Schmidt and Lee, 2019) and represents the time it takes to transform the electrical activity from the central drive to muscle force.

The anticipatory period is the time prior to the presentation of a stimulus (Niemi and Näätänen, 1981; Schmidt and Lee, 2019). The characteristics

1 - Department of Applied Physiology and Kinesiology, University of Florida, Gainesville, FL, USA, 32611. 
of the anticipatory period influence the speed of the reaction (Schmidt and Lee, 2019). Previous studies investigated the effect of the anticipatory period on RT, by manipulating the length of the anticipatory period (Drazin, 1961; Niemi and Näätänen, 1981; Olivier and Rival, 2002; Sanders, 1975), the warning cue provided (Perchet and Garcia-Larrea, 2005; Schmidt and Lee, 2019), the presence of a stressor or a distracting environment (Fisk et al., 2002; Lorist et al., 2002; Ninio and Kahneman, 1974), the amount of force produced (Temprado et al., 2015; Zijdewind et al., 2006), and the difficulty of the anticipatory task (Strayer and Johnston, 2001). For example, the exertion of higher forces during the anticipatory period results in longer RTs (Temprado et al., 2015; Zijdewind et al., 2006). Specifically, RT was longer when participants produced $50 \%$ of their maximum voluntary contraction (MVC) compared with 10\% MVC during a steady force tracking task (Temprado et al., 2015). Moreover, Strayer and Johnston (2001) manipulated the difficulty of the anticipatory period by comparing RT after a single and a dual task. The single task required participants to align a joystick to a moving target (pursuit tracking task), whereas the dual task required participants to perform the same pursuit tracking task while engaging in conversation. They found that greater task difficulty during the anticipatory period (dual task condition) resulted in longer RTs (Strayer and Johnston, 2001). Although these findings demonstrate the importance of the anticipatory period to RT, it remains unknown whether the strategy used during the anticipatory period alters the speed of the reaction.

The purpose of this study, therefore, was to determine whether the speed of the reaction differs between a steady and a dynamic anticipatory strategy. We manipulated the anticipatory period by asking participants to either maintain (steady) or oscillate (dynamic) their force output prior to a visual stimulus. We hypothesized that the dynamic anticipatory strategy would result in a longer RT due to the variable levels and direction of force at which the reaction can occur.

\section{Methods}

\section{Participants}

Twenty-two young adults $(21.0 \pm 2.2$ yrs,
13 women) volunteered to participate in this study. All participants reported being healthy without any known neurological or orthopedic disorders. On average participants had a BMI of $22.3 \pm 2.4$ and a MOCA score of $28.1 \pm 1.5$ (Bourgeois-Marcotte et al., 2015). All participants were right handed and right footed as assessed with the Edinburgh Handedness Inventory (Oldfield, 1971) and the Waterloo Footedness Questionnaire (Elias and Bryden, 1998), respectively. The Institutional Review Board at the University of Florida approved the procedures, and participants signed a written informed consent form before in the beginning of the study.

\section{Experimental approach}

Participants performed one testing session that lasted $\sim 2$ hours. This session involved performing a reaction time task with a steady and a dynamic anticipatory force control strategy. For each participant, we randomized the order of the anticipatory strategy to control for a possible task order effect. Participants performed the following: 1) familiarization with the experimental procedure, which included a verbal explanation and demonstration of the reaction time task; 2) maximum voluntary contraction (MVC) with ankle dorsiflexion; 3) 15 trials of the steady reaction time task and 15 trials of the dynamic reaction time task (randomized order); 4) repetition of the MVC task.

\section{Experimental arrangement}

Experimental setup and apparatus. Each participant sat comfortably in an upright position and faced a 32-inch monitor (SyncMasterTM 320MP-2, Samsung Electronics America, Ridgefield Park, NJ, USA) that was located $1.25 \mathrm{~m}$ away at the eye level. The monitor displayed the targeted force, the force produced by ankle dorsiflexion, and the stimulus using a customwritten program in Matlab ${ }^{\circledR}$ (Math WorksTM Inc., Natick, MA, USA). All participants affirmed that they could see the display clearly. The participants' left hip joint was at $\sim 90^{\circ}$ flexion and $\sim 10^{\circ}$ abduction, while the knee joint was at $\sim 90^{\circ}$ flexion. The left foot rested on a customized foot device with an adjustable footplate and was secured by straps over the metatarsals to ensure a secure position and isolated dorsiflexion of the ankle (Figure 1A). The initial ankle position was $\sim 90^{\circ}$ of ankle dorsiflexion. All participants 
performed the reaction time tasks with their nondominant foot (i.e. the left) so that the task would be more novel than with the dominant limb (Sainburg, 2002).

Force measurements. We quantified the force exerted during the MVC and reaction time task with a force transducer (Model MB-100, Interface, Scottsdale, AZ, USA) placed in parallel with the direction of the customized foot device. The ankle force signals were amplified 100 times (Bridge-8, World Precision Instruments), sampled at $1,000 \mathrm{~Hz}$ with a NI-DAQ card (model USB6210, National Instruments), and stored on a personal computer.

EMG measurements. To identify the onset of muscle activity during the reaction time task, we recorded the muscle activity of the primary ankle dorsiflexor (tibialis anterior; TA) with surface electromyography (EMG) (Bagnoli EMG system; Delsys Inc., Boston, MA, USA). We placed the recording electrodes on the skin and in line with the muscle fibers of the TA at the proximal $1 / 3$ between the head of the fibula and the medial malleolus. We placed the reference electrode over the patella. The EMG signals were amplified 1000 times, sampled at $1000 \mathrm{~Hz}$ with a data acquisition (DAQ) card (Model USB6210, National Instruments, Austin, TX, USA), and stored on a personal computer.

MVC task. We identified the MVC for ankle dorsiflexion before and after the reaction time tasks. Participants increased their ankle dorsiflexion force to their maximum and maintained it for 3 s. Participants exerted 3-5 MVCs until two MVC values were within 5\% of each other. There was one minute of rest between trials to prevent fatigue. We repeated the MVC task at the end of the experiment to assess whether the experimental task induced fatigue.

Reaction time task. The reaction time task consisted of either a steady or a dynamic anticipatory period and the reaction to an unanticipated visual stimulus. During the steady anticipatory period, we asked the participants to perform a steady isometric force-tracking task that required them to maintain a target force $(15 \%$ MVC) with ankle dorsiflexion as accurately and as consistently as possible. During the dynamic anticipatory period, we asked the participants to increase and decrease their force in a sinusoidal pattern within a target force area (15 $\pm 5 \%$ MVC).
In both cases, we displayed the target area as two red horizontal lines over a white background in the middle of the monitor and the force produced by the participant as a blue line progressing with time from left to right (Figure 1B); and the visual gain was kept steady at $1.2^{\circ}$ (visual angle) (Vaillancourt et al., 2006).

The anticipatory period finished when an unanticipated visual stimulus appeared in the middle of the screen. The stimulus consisted of a transient change in background color, from white to green, that lasted for one second (Figure 1B). We instructed the participants to respond to the stimulus as quickly as possible by increasing their ankle dorsiflexion with enough force to show the reaction clearly. Each trial lasted 37 s. Participants performed 15 trials for each condition. For 12 out of the 15 trials, we randomly presented the stimulus between seconds 22 and 25 . For the other three trials, we presented the stimulus at second 13.5. We did this to prevent participants from predicting an occurrence of the stimulus around the $22 \mathrm{~s}$ mark. Moreover, we manually inspected the data and classified each dynamic trial based on the location of the force onset in relation to the sinusoidal force pattern: middle ascending (MA), peak $(\mathrm{P})$, middle descending $(\mathrm{MD})$, or trough $(\mathrm{T})$ of the sinusoidal force trace (Figure 1C). On average, from the 12 analyzed trials per participant, $3 \pm 1$ trials occurred at each of the four dynamic categories.

\section{Data analysis}

We analyzed the force and EMG signals using a custom-written program in Matlab®. In the analysis, we included only the 12 trials in which the stimulus occurred from 22 to $25 \mathrm{~s}$. Prior to data analysis, the program low-pass filtered the raw force signal at $20 \mathrm{~Hz}$ with a fourth-order (bidirectional) Butterworth filter and detrended it. Detrending the force signal removed the linear trend from the data and eliminated any drift. We identified force onset as the first time point in which the force exceeded 2 SD of the mean force around the stimulus onset (i.e. $200 \mathrm{~ms}$ before the stimulus and $100 \mathrm{~ms}$ after the stimulus). Moreover, the EMG signals were high-passed filtered at $10 \mathrm{~Hz}$, rectified, and low-pass filtered at $6 \mathrm{~Hz}$. We used this filter to identify the onset of EMG associated with the reaction to the stimulus. We identified EMG onset as the first time point in which the low-passed filtered EMG was greater 
than $2 \mathrm{SD}$ of the mean EMG around the stimulus onset (i.e. $200 \mathrm{~ms}$ before the stimulus and $100 \mathrm{~ms}$ after the stimulus) (Figure 2A).

Strength. We quantified strength with the force produced during the MVC task. From the MVC trials that were within $5 \%$ of each other, we selected the trial of highest force as a representation of the participant's maximal strength.

Speed of the response. We quantified RT as the average (across the 12 analyzed trials) temporal interval from the stimulus onset to the force onset (Figure 2A). Additionally, we decomposed RT into premotor time and electromechanical delay (EMD). We quantified premotor time as the time interval from the stimulus onset to the EMG onset, and EMD as the time interval from the EMG onset to the force onset (Figure 2A).

\section{Statistical Analysis}

We used a paired t-test to compare participant's strength before and after the tasks as well as RT between the steady and the dynamic conditions. We used repeated measures ANOVA with non-orthogonal planned contrast (simple) to compare the RT of the steady condition (reference group in the planned contrast) to each of the categories of the dynamic condition. We then used a paired t-test to compare premotor time and EMD between the steady and the middle descending conditions. We focused on the middle descending condition because it resulted in a longer RT even though participants exerted a similar anticipatory force level to the steady condition ( $15 \%$ MVC). We performed all statistical analyses using the IBM statistics 24.0 statistical packages (IBM Inc., New York). The alpha level was $p=0.05$. Data are reported as mean \pm SD within the text and as mean \pm standard error of the mean (SEM) in the figures.

\section{Results}

Strength and fatigue. MVC forces before and after the reaction time tasks were similar among participants (before: $200.8 \pm 58.5 \mathrm{~N}$ vs. $203.7 \pm 52.5 \mathrm{~N}, \mathrm{t}=-0.456, p=0.65)$. These results suggest that the experimental protocol did not induce fatigue and, therefore, fatigue did not play a role in the results below.

Reaction time. To assess whether a steady or a dynamic anticipatory strategy affected reaction time, we compared participants' RT between the steady and dynamic condition. We found that when participants performed a steady force control during the anticipatory period, they exhibited a shorter RT than when they performed a dynamic force control (Steady: $478.8 \pm 49.4 \mathrm{~ms}$, Dynamic: $506.6 \pm 63.0 \mathrm{~ms}, \mathrm{t}=-2.57, p<0.02$; Figure 3A).

Although the force level was similar between the steady and the middle ascending or descending conditions of the dynamic task $(\sim 15 \%)$, the direction of force varied between these conditions. On the other hand, the force level was greater at the peak $(\sim 20 \%)$ and lesser at the trough $(\sim 10 \%)$ of the dynamic compared with the steady task $(\sim 15 \%)$. Thus, we examined the effect of the variable levels and direction of force by comparing the steady RT to that of each category of the dynamic strategy. We found that RT was similar between the steady and the middle ascending (Steady: $478.8 \pm 49.4 \mathrm{~ms}$, Middle Ascending: $455.6 \pm 83.6 \mathrm{~ms}, \mathrm{~F}_{1,19}=0.235, p=0.63$; Figure 3B) conditions. However, RT was longer at the peak $\left(531.0 \pm 103.4 \mathrm{~ms}, \mathrm{~F}_{1,19}=7.00, p<0.02\right.$; Figure 3B), the trough $\left(509.7 \pm 76.6 \mathrm{~ms}, \mathrm{~F}_{1,19}=6.34\right.$, $p=0.02$; Figure $3 \mathrm{~B})$, and the middle descending $\left(534.4 \pm 76.6 \mathrm{~ms}, \mathrm{~F}_{1,19}=11.27, p<0.01\right.$; Figure 3B) relative to the steady condition $(478.8 \pm 49.4 \mathrm{~ms})$.

Premotor time. We found that premotor time was similar between the steady $(421.8 \pm 48.1$ $\mathrm{ms})$ and the trough $\left(432.5 \pm 73.6 \mathrm{~ms}, \mathrm{~F}_{1,19}=1.54, p=\right.$ 0.23 ; Figure $4 \mathrm{~A}$ ), as well as the steady and the middle ascending $\left(385.8 \pm 81.8 \mathrm{~ms}, \mathrm{~F}_{1,19}=1.99, p=\right.$ 0.18 ; Figure 4A) conditions. However, we found that, compared with the steady $(421.8 \pm 48.1 \mathrm{~ms})$, premotor time was longer at the peak (465.2 \pm $102.1 \mathrm{~ms}, \mathrm{~F}_{1,19}=4.30, p=0.05$; Figure $4 \mathrm{~A}$ ) and the middle descending conditions $(459.3 \pm 76.6 \mathrm{~ms}$, $\mathrm{F}_{1,19}=4.82, p=0.04$; Figure 4A).

EMD. We found that the EMD under the steady condition $(57.9 \pm 6.2 \mathrm{~ms})$ was shorter than under the trough $\left(77.2 \pm 8.8 \mathrm{~ms}, \mathrm{~F}_{1,19}=75.8, p<\right.$ 0.01 ; Figure $4 \mathrm{~B})$, the middle ascending $(69.7 \pm 12.7$ ms, $F_{1,19}=20.6, p<0.01$; Figure $\left.4 \mathrm{~B}\right)$, the peak $(67.8$ $\pm 12.9 \mathrm{~ms}, \mathrm{~F}_{1,19}=9.32, p<0.01$; Figure $\left.4 \mathrm{~B}\right)$, and the middle descending $\left(75.1 \pm 12.7 \mathrm{~ms}, \mathrm{~F}_{1,19}=44.8, p<\right.$ 0.01 ; Figure 4B) conditions. 
A

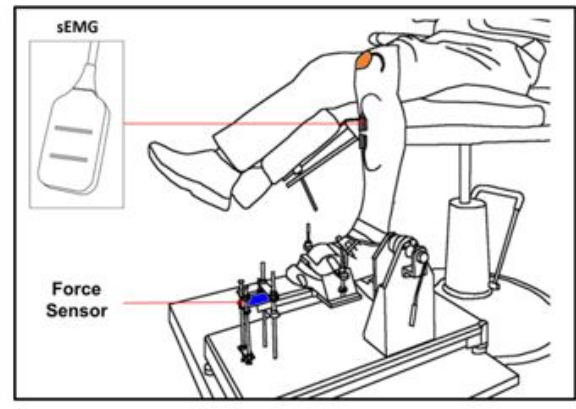

B
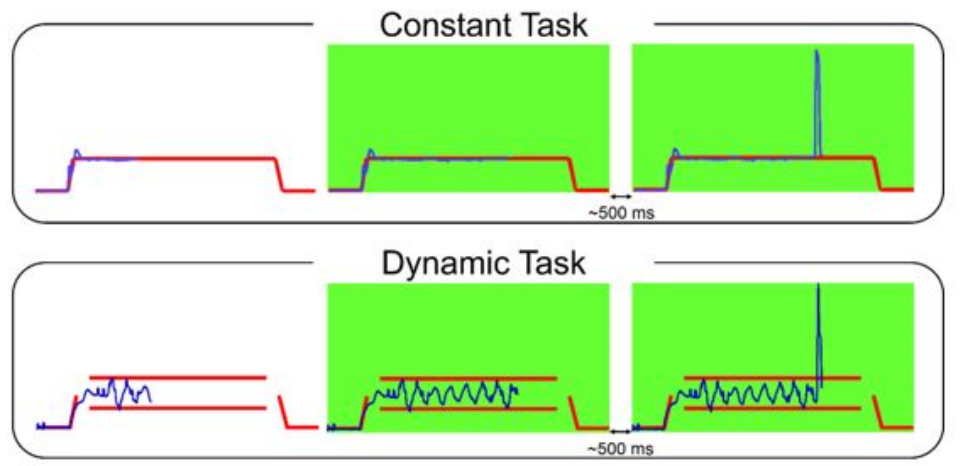

C
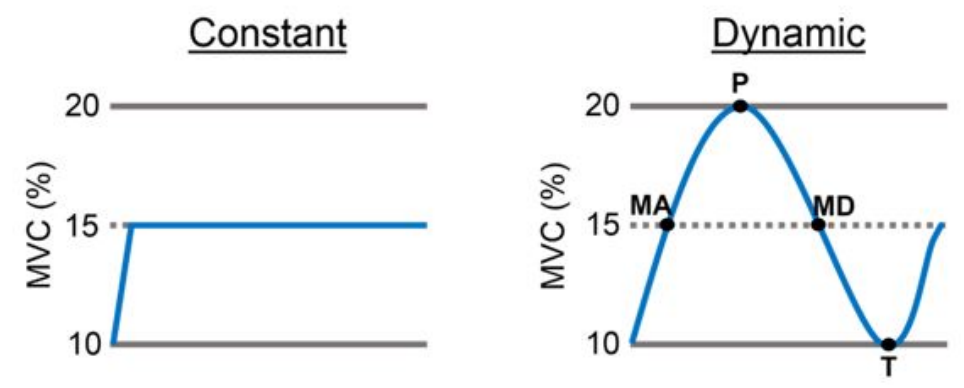

Figure 1

Task Procedures. (A) Schematic drawing of the experimental set up and arrangement of the left foot. Participants performed a reaction time task with ankle dorsiflexion. (B) The left panels show the anticipatory strategy (type of force control before the stimulus), the middle panels show the moment when the stimulus first occurs, and the right panels show the reaction of the participant. (C) The left panel shows the steady condition in which participants had to maintain their force at $15 \%$ MVC. The right panel shows the dynamic condition in which participants had to oscillate their force at $15 \pm 5 \%$ MVC. We classified each dynamic trial based on whether the reaction occurred at middle ascending $(M A)$, peak $(P)$, middle descending $(M D)$, or the trough $(T)$ of the sinusoidal force trace. 


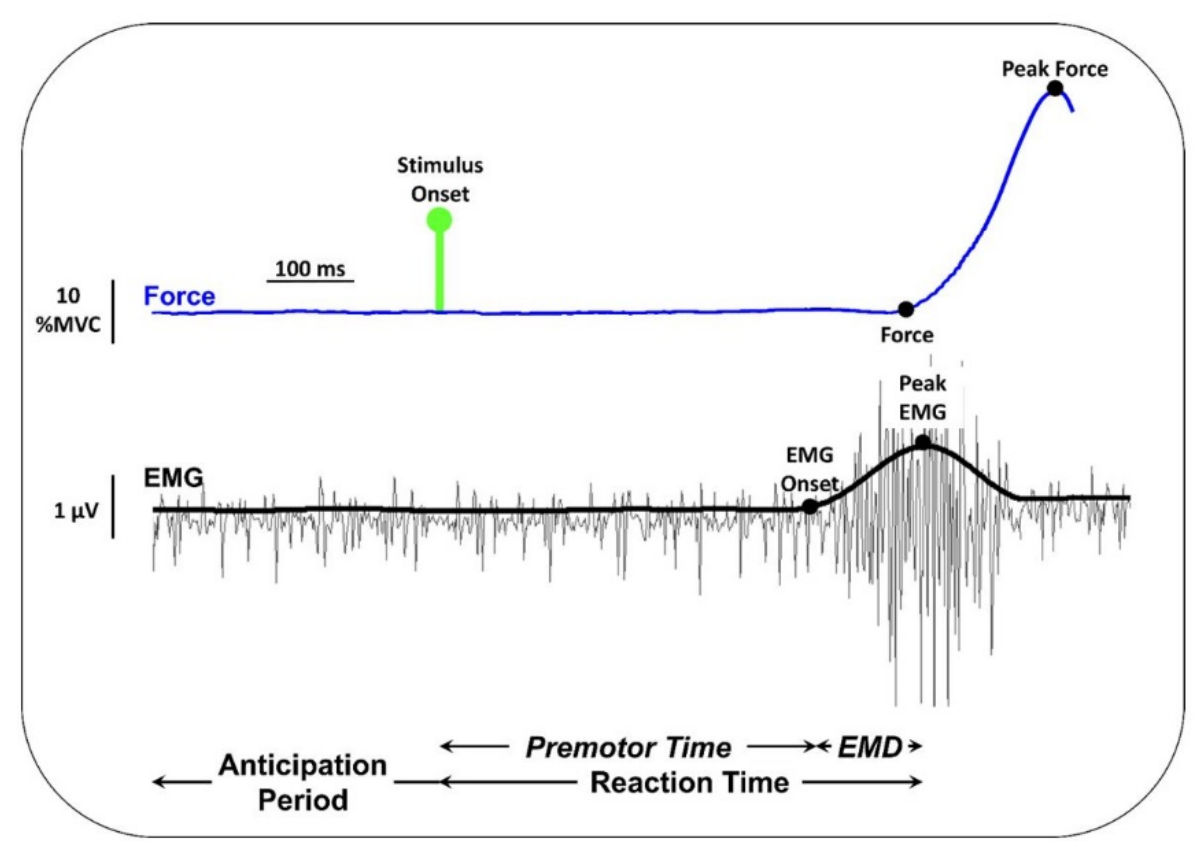

Figure 2

Variables of Interest. Graphical representation of the quantification of the different variables of interest.

A

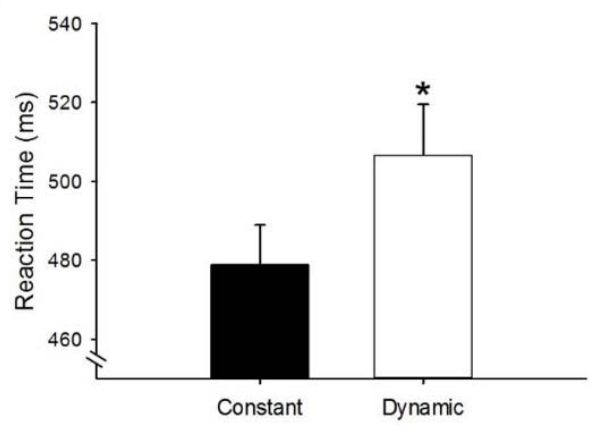

B

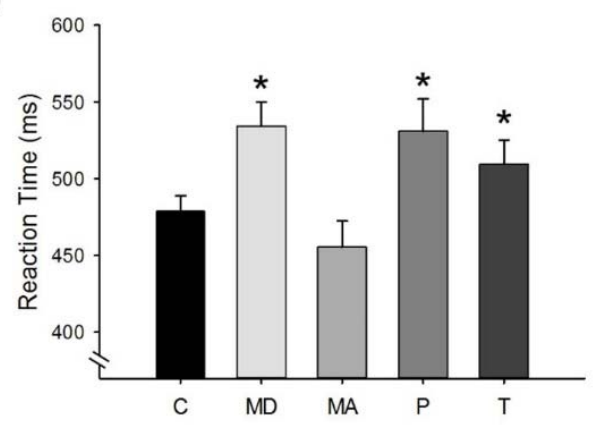

Figure 3

Reaction Time Results. (A) Reaction time was shorter following a steady than a dynamic anticipatory strategy. (B) Reaction time was longer when the response occurred at the middle descending $(M D)$, peak $(P)$, and trough $(T)$ compared with the steady strategy. Moreover, when the reaction occurred at the middle ascending $(M A)$, the RT was similar to that of the steady strategy. ${ }^{*}$ Significantly different from the steady anticipatory strategy. 
A

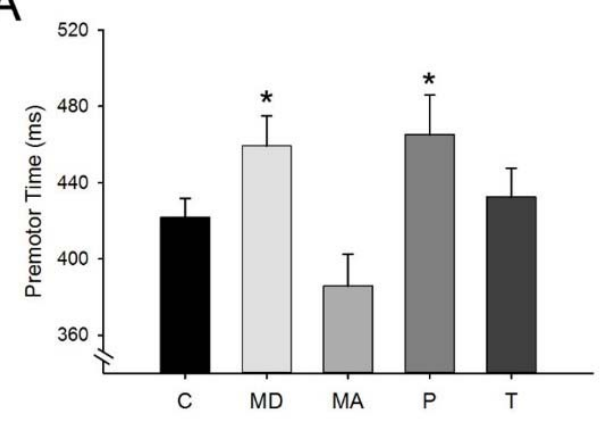

B

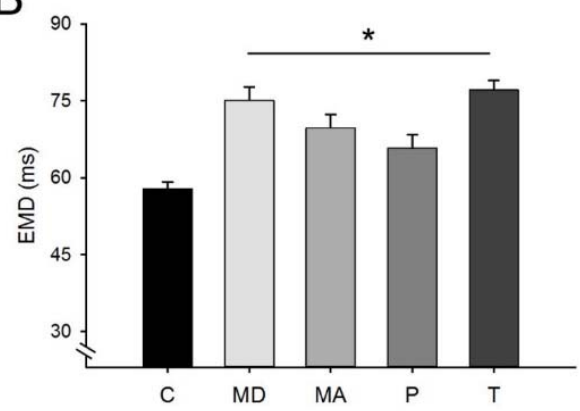

Figure 4

Component Results. (A) Premotor time was longer when the response occurred at the middle descending $(\mathrm{MD})$ and peak $(\mathrm{P})$ categories of the dynamic strategy compared with the steady strategy. Moreover, when the reaction occurred at the middle ascending (MA) and trough (T), the RT was similar to that of the steady strategy. (B) EMD was longer in every category of the dynamic strategy compared with the steady strategy. ${ }^{*}$ Significantly different from the steady anticipatory strategy.

\section{Discussion}

The purpose of this study was to determine if a steady and a dynamic anticipatory strategy result in different RTs. The dynamic anticipatory strategy resulted in a longer RT due to the variable levels and directions of force at which the response occurred. These results provide novel evidence that the strategy used to anticipate a visual stimulus influences the speed of the reaction.

\section{Reaction Time}

We provide novel evidence that anticipating a visual stimulus with a dynamic strategy results in a longer response than anticipating with a steady strategy. It is possible that the constantly changing levels and directions of force during the dynamic anticipatory strategy underlie the observed differences in RT. Interestingly, our findings show that during a dynamic anticipatory strategy, the variable directions, but not the variable levels, of force at which the response occurs lengthen RT.

Force level. During our dynamic force control task, we asked participants to oscillate their force output between 10 and $20 \%$ of their MVC. Since we randomly assigned the visual stimulus, participants could respond at any force level within this range. Thus, we examined how the variable force levels contributed to the difference in RT between the steady and the dynamic condition. To this end, we classified the trials of the dynamic condition based on the level of force at the moment of the response (Figure 1C). We found that RT was similar between the steady and the middle ascending conditions, but longer during the middle descending, the peak, and the trough conditions. Since the steady, the middle ascending, and the middle descending conditions all were at similar force levels (i.e. 
$\sim 15 \% \mathrm{MVC}$ ), our results suggest that the variable force levels at the moment of the response cannot fully explain the observed RT differences between the two anticipatory strategies.

Force direction. During the dynamic anticipatory strategy, direction of force is also continuously changing. Decreasing the amount of force is a more difficult process to control than increasing the force (Park et al., 2016; Spiegel et al., 1996). In addition, evidence suggests that more difficult anticipatory strategies result in longer RT (Strayer and Johnston, 2001). It is possible, therefore, that the direction of force at the time of response initiation influences RT. Thus, we compared RT under the steady condition to the RT under the middle ascending (MA) and the middle descending (MD) conditions of the dynamic anticipatory strategy. This allowed us to control the force level at which the response occurred ( 15 MVC), while comparing RT when the anticipatory force was maintained, increased or decreased. We found that, compared with the steady, the middle ascending condition resulted in a similar RT, whereas the middle descending condition resulted in a longer RT. Thus, our results suggest that the force direction at the time of response initiation influences RT.

\section{Premotor time and EMD}

RT consists of two components: premotor time and electromechanical delay (EMD) (Schmidt and Lee, 2019). Accordingly, our results prompted the following question: How are premotor time and EMD affected by the levels and directions of force at the time of response initiation?

Premotor time. We found that only premotor time during the peak and middle descending part of the dynamic anticipatory strategy was longer relative to the steady anticipatory strategy. This finding suggests that differences in the force level cannot account for the observed differences in premotor time. Premotor time represents the time it takes for the nervous system to identify the stimulus, select and plan the response (i.e. motor planning) (Botwinick and Thompson, 1966; Schmidt and Lee, 2019). Since the stimulus and the response required are the same for all categories, one may hypothesize that the time required to identify the stimulus and select the response should be similar in all categories. In contrast, we hypothesized that the anticipatory force directions at which we presented the stimulus would affect the time it takes to plan the response.

Along these lines, we recently examined whether the location of a visual stimulus during a sinusoidal reaction time task affected RT (Delmas et al., 2018). We compared RT after the stimulus appeared at the peak and at the trough of the anticipatory sinusoid. At the peak, the motor plan was to decrease force by reducing net muscle activity in accordance with tracing the sinusoidal task. However, the response requirement was to increase force by increasing net muscle activity, which required a change in the motor plan. Thus, when the stimulus appeared at the peak, it perturbed the motor plan of the anticipatory period. At the trough, the motor plan was to increase force in accordance with the task and participants had to simply continue increasing force to react. Thus, when the stimulus appeared at the trough, the motor plan for the anticipatory and response phases was compatible and no perturbation to the anticipatory motor plan occurred. This manipulation allowed us to determine that the location of the stimulus challenged the motor planning process and lengthened RT (Delmas et al., 2018).

In the present study, the anticipatory motor plan during the steady task was to maintain the force output at the same level, whereas during the dynamic task, the plan was to increase and decrease the force output. In addition, the motor plan for the response in both tasks was to increase the force output. If the stimulus appears when the anticipatory plan is to increase or maintain the force, then the motor plans of the anticipation and the response do not oppose each other. On the other hand, if the stimulus appears when the anticipatory plan is to decrease the force, then the anticipatory and response motor plans oppose each other. Thus, opposition between the anticipatory and response plans requires a change in the motor plan and results in longer premotor times.

Our results are in line with this idea. After dividing the dynamic task into different categories, we found that the premotor time was significantly longer when the response occurred at the peak or middle descending categories relative to the steady condition. For the reaction to occur at the peak, the stimulus appeared at the middle ascending, while for the reaction to occur 
at the middle descending, the stimulus appeared at the peak. In these situations, the anticipatory and the response motor plans opposed each other, which challenged the motor planning process, and thus lengthened RTs. On the other hand, the premotor time was similar when the response occurred at the trough or middle ascending categories relative to the steady condition. For the reaction to occur at the trough, the stimulus occurred at the middle descending, while for the reaction to occur at the middle ascending, the stimulus appeared at the trough. In these situations, the anticipatory and the response motor plans did not oppose each other, which did not challenge the motor planning process, and thus resulted in similar RTs. Therefore, our results provide additional support to the idea that challenging the motor planning process results in longer reaction times. We did so by demonstrating that the variable directions of force (during the dynamic anticipatory strategy) at the time of the response affected the speed of the reaction. In addition, we demonstrated that a steady anticipatory strategy did not challenge the motor planning process.

EMD. We found that the EMD was longer in every category of the dynamic condition compared with the steady condition. EMD represents the time it takes for the nervous system to convert the electrical activity from the central nervous system into muscle force (Botwinick and Thompson, 1966; Schmidt and Lee, 2019). The modulation of the motor neuron pool, the contractile components of the muscle, and muscle-tendon stiffness can influence the length of the EMD (Cavanagh and Komi, 1979; Grosset et al., 2009; Norman and Komi, 1979; Waugh et al., 2013). The longer EMD under the dynamic condition could come from an altered modulation of the motor units between the two conditions. During the dynamic (oscillatory) anticipation strategy, the number of motor units and their firing rates must increase and decrease to achieve the sinusoidal force (Enoka and Duchateau, 2017). In contrast, during the steady anticipation strategy the number of recruited motor units and their firing rates remain relatively stable (Enoka and Duchateau, 2017). Alternatively, the mechanical properties of the muscle are different for the two strategies. For example, the length of the series elastic component remains relatively unchanged for the steady strategy, but changes during the dynamic strategy. The time it takes to stretch the series elastic component contributes significantly to EMD (Muraoka et al., 2004), and thus it could contribute to the lengthening of the EMD under the dynamic condition.

\section{Limitations}

In this study, we only investigated the effect of a steady or a dynamic anticipatory strategy on RT. However, when attempting to generalize our findings to sports or any other daily living activity, one must take into account a myriad of variables that interplay with each other. For example, in soccer, goalkeepers may decide to sacrifice some speed as a way to increase their accuracy or to distract the kicker. Future studies should investigate the effect of these anticipatory strategies on accuracy and other sport related variables. In addition, future studies should use multi motor unit recording and high-resolution ultrasonography, to determine the contribution of neural and mechanical factors to the lengthening of RT during the anticipatory strategy. Finally, we only analyzed healthy young adults. Further research is required to examine the effects of each anticipatory strategy on the RT of older adults and individuals with neurological diseases.

\section{Conclusion}

Taken all together, we provide novel evidence that RT is shorter following a steady anticipatory strategy than a dynamic one. We propose that the longer RTs after a dynamic anticipatory strategy arise from the variable force directions at which the response can occur which challenge the motor planning process. In conclusion, a steady anticipatory strategy appears to be advantageous for tasks in which the speed of $\mathrm{RT}$ is essential. 


\section{References}

Botwinick J, Thompson LW. Premotor and motor components of reaction time. J Exp Psychol, 1966; 71: 9-15

Bourgeois-Marcotte J, Flamand-Roze C, Denier C, Monetta L. LAST-Q: Adaptation and normalisation in Quebec of the Language Screening Test. Rev Neurol (Paris), 2015; 171: 433-436

Cavanagh PR, Komi P V. Electromechanical delay in human skeletal muscle under concentric and eccentric contractions. Eur J Appl Physiol Occup Physiol, 1979; 42: 159-163

Delmas S, Casamento Moran A, Park SH, Yacoubi B, Christou EA. Motor Planning Perturbation: Muscle Activation and Reaction Time. J Neurophysiol, 2018: jn.00323.2018

Drazin DH. Effects of foreperiod, foreperiod variability, and probability of stimulus occurrence on simple reaction time. J Exp Psychol, 1961; 62: 43

Elias LJ, Bryden MP. Footedness is a better predictor of language lateralisation than handedness. Laterality, 1998; 3: 41-51

Enoka RM, Duchateau J. Rate Coding and the Control of Muscle Force. Cold Spring Harb Perspect Med, 2017: a029702

Fisk GD, Owsley C, Mennemeier M. Vision, attention, and self-reported driving behaviors in communitydwelling stroke survivors. Arch Phys Med Rehabil, 2002; 83: 469-477

Grosset JF, Piscione J, Lambertz D, Pérot C. Paired changes in electromechanical delay and musculotendinous stiffness after endurance or plyometric training. Eur J Appl Physiol, 2009; 105: 131-139

Lorist MM, Kernell D, Meijman TF, Zijdewind I. Motor fatigue and cognitive task performance in humans. J Physiol, 2002; 545: 313-319

Muraoka T, Muramatsu T, Fukunaga T, Kanehisa H. Influence of tendon slack on electromechanical delay in the human medial gastrocnemius in vivo. J Appl Physiol, 2004; 96: 540-544

Niemi P, Näätänen R. Foreperiod and simple reaction time. Psychol Bull, 1981; 89: 133

Ninio A, Kahneman D. Reaction time in focused and in divided attention. J Exp Psychol, 1974; 103: 394-399

Norman RW, Komi P V. Electromechanical delay in skeletal muscle under normal movement conditions. Acta Physiol Scand, 1979; 106: 241-8

Oldfield RC. The assessment and analysis of handedness: the Edinburgh inventory. Neuropsychologia, 1971; 9: 97-113

Olivier I, Rival C. Foreperiod duration and motor preparation during childhood. Neurosci Lett, 2002; 319: $125-128$

Park SH, Kwon M, Solis D, Lodha N, Christou EA. Motor control differs for increasing and releasing force. J Neurophysiol, 2016: jn 007152015

Perchet C, Garcia-Larrea L. Learning to react: Anticipatory mechanisms in children and adults during a visuospatial attention task. Clin Neurophysiol, 2005; 116: 1906-1917

Sainburg RL. Evidence for a dynamic-dominance hypothesis of handedness. Exp Brain Res, 2002; 142: 241

Sanders AF. The foreperiod effect revisited. Q J Exp Psychol, 1975; 27: 591-598

Schmidt RA, Lee T. Motor control and learning: A behavioral emphasis. Sixth Edition. Champaign, IL 61825-5076: Human kinetics; 2019

Spiegel KM, Stratton J, Burke JR, Glendinning DS, Enoka RM. The influence of age on the assessment of motor unit activation in a human hand muscle. Exp Physiol, 1996; 81: 805-819

Strayer DL, Johnston WA. Driven to Distraction: Dual-Task Studies of Simulated Driving and Conversing on a Cellular Telephone. Psychol Sci, 2001; 12: 462-466

Temprado J-JJ, Vieluf S, Bricot N, Berton E, Sleimen-Malkoun R, Peng C. Performing isometric force control in combination with a cognitive task: A multidimensional assessment. PLoS One, 2015; 10: e0142627 
Vaillancourt DE, Haibach PS, Newell KM. Visual angle is the critical variable mediating gain-related effects in manual control. Exp Brain Res, 2006; 173: 742-750

Waugh CM, Korff T, Fath F, Blazevich AJ. Rapid force production in children and adults: Mechanical and neural contributions. Med Sci Sports Exerc, 2013; 45: 762-771

Zijdewind I, van Duinen H, Zielman R, Lorist MM. Interaction between force production and cognitive performance in humans. Clin Neurophysiol, 2006; 117: 660-667

\section{Corresponding author:}

\section{Evangelos A. Christou, Ph.D.}

Department of Applied Physiology and Kinesiology

University of Florida

Room 100 Florida Gym Stadium RD

Gainesville - FL 32611-8205, USA

Phone: 352-294-1719

FAX: 352-392-5262

E-mail: eachristou@ufl.edu 